\title{
FORMULATION OF NANOPARTICLES OF EPROSARTAN MESYLATE FOR THE BETTER DRUG DELIVERY BY IMPROVING SOLUBILITY
}

\author{
UPASANA YADAV ${ }^{1 *}$, NUZHAT HUSAIN ${ }^{2}$, QAMAR REHMAN ${ }^{3}$
}

${ }^{1}$ Department of Biotechnology, School of Applied Sciences, Amity University, Lucknow, Uttar Pradesh, India. ${ }^{2}$ Department of Pathology, Dr. Ram Manohar Lohia Institute of Medical Sciences, Lucknow, Uttar Pradesh, India. ${ }^{3}$ Department of Biotechnology, Amity University, Lucknow, Uttar Pradesh, India. Email: profupasana.yadav@gmail.com

Received: 26 April 2018, Revised and Accepted: 13 June 2018

ABSTRACT

Objective: In the present study, we have made an attempt to the developed formulation of nanoparticles (NPs) of eprosartan mesylate (EM) incorporated in carboxymethyl chitosan using reverse micelle technique for the better drug delivery by improving solubility.

Methods: The NPs size and morphology were investigated by high-resolution transmission electron microscopy and field emission scanning electron microscopy, respectively. The physical and chemical aggregation state of eprosartan was analyzed using ultraviolet spectroscopy, and Fourier transforms infrared spectroscopy.

Results: To increase the solubility of eprosartan by reverse micelle technique of the drug through polymeric NPs is an alternative efficient, option for increasing the solubility. Eprosartan nanosuspension was successfully formulated for dissolution and bioavailability enhancement of the drug. The percentage drug release pattern of both formulations was compared against that of pure drug. It shows that in 10 min $39 \%$ and $17 \%$ of drug was released from the NPs made by RM method and microemulsion method, respectively, as compared to that of $1.3 \%$ of the pure drug. In 50 min almost more than half $51 \%$ of the drug was released from NPs by microemulsion method whereas only $2.5 \%$ of the drug was released from NPs containing the pure drug. In $120 \mathrm{~min} 67 \%$ of the drug was released from NPs by microemulsion method whereas only $5.8 \%$ of drug release was shown by NPs with the pure drug. We are paying attention on evaluating the influence of particle size and crystalline state on the in vitro performance of eprosartan.

Conclusion: In summary, we have developed a new approach toward the delivery of poorly water-soluble drug eprosartan by reverse micellar method. The particle size of NPs obtained by the reverse micellar method was significantly reduced as compared to the other method.

Keywords: Eprosartan, Nanoparticle, Carboxymethyl chitosan, Reverse micellar method.

(C) 2018 The Authors. Published by Innovare Academic Sciences Pvt Ltd. This is an open access article under the CC BY license (http://creativecommons. org/licenses/by/4. 0/) DOI: http://dx.doi.org/10.22159/ajpcr.2018.v11i10.26915

\section{INTRODUCTION}

Eprosartan mesylate (EM) is an "angiotensin II receptor antagonist" which is used for treating high blood pressure. As compared to other angiotensin II receptor antagonists, eprosartan is reported to be better tolerated than enalapril [1]. EM antagonizes the effect of angiotensin II (vasoconstriction and aldosterone secretion) by blocking the angiotensin II receptor in vascular smooth muscle and the adrenal gland producing decreased blood pressure [2]. It is a BCS Class II, insoluble in water, antihypertensive drug having $13 \%$ oral bioavailability $[3,4]$. It has been observed and proved that the bioavailability of poorly soluble drug improves by nanonization, following the production of nanoparticles (NPs) by Sucker et al. in 1980, the nanosizing has gained a lot of attention [5-14]. According to Noyes Whitney equation, it has been observed experimentally that the reduction in particle size of a sparingly soluble material results in an increased rate of solution. Noyes Whitney is regularly used to describe the process of dissolution of solid drugs:

$\mathrm{Dm} / \mathrm{dt}=\mathrm{DA}\left(\mathrm{C}_{\mathrm{s}}-\mathrm{C}\right) / \mathrm{h}$

Where the rate of change of mass dissolved $(\mathrm{m})$ with time $(\mathrm{t})$ is related to the diffusion coefficient (D) through a static layer of liquid of thickness $\mathrm{h}$, and $\mathrm{C}_{\mathrm{s}}$ is the equilibrium solubility and the amount dissolved at time $\mathrm{t}$ (C) in that an increase in the surface area of a drug will result in a more rapid dissolution process, particularly under sink conditions (where $\mathrm{C}$ $<<\mathrm{C}_{\mathrm{s}}$ ) [15].

The concept of reducing particle size to nanorange and comparing two different methods for the preparation of NPs has been explored in this experiment. Ionic gelation method and reverse micellar method were compared for the preparation of NPs, and their effect on particle size and hence solubility has been determined. Chitosan NP prepared by ionotropic gelation technique was first reported by Calvo and has been widely examined and developed by Janes. The electrostatic interaction between amine group of chitosan and negatively charge group of polyanion such as tripolyphosphate is the mechanism on which formation of chitosan NP is based. This technique offers a simple and mild preparation method in the aqueous environment. First, chitosan can be dissolved in acetic acid in the absence or presence of stabilizing agents, such as poloxamer, which can be added in the chitosan solution before or after the addition of polyanion. Polyanion or anionic polymers were then added, and NPs were spontaneously formed under mechanical stirring at room temperature. The size and surface charge of particles can be modified by varying the ratio of chitosan and stabilizer. Chitosan NP prepared by microemulsion technique was first developed by Maitra. This technique is based on the formation of chitosan NP in the aqueous core of reverse micellar droplets and subsequently cross-linked through glutaraldehyde. This technique offers a narrow size distribution of $<100 \mathrm{~nm}$, and the particle size can be controlled by varying the amount of glutaraldehyde that alters the degree of cross-linking. Nevertheless, some disadvantages exist such as the use of organic solvent, time-consuming preparation process, and complexity in the washing step [16].

\section{METHODS}

Drugs and chemical

Chitosan (CS) (Mw = 100,000-300,000, deacetylation degree $\geq 90 \%$ as determined by free amine groups) and monochloroacetic acid were 
acquired from Sigma-Aldrich. Calcium chloride, isopropanol, methanol, and ethanol were obtained from Merck India; water was Millipore water. 3-(4,5-dimethylthiazol-2-yl)-2,5-diphenyltetrazolium bromide and EM were purchased from Sigma-Aldrich, USA. Dimethyl sulfoxide and $\mathrm{N}, \mathrm{N}$-dimethylformamide were obtained from Merck India and purified by vacuum distillation. Fetal bovine serum and minimum essential medium were procured from Hyclone, USA, and HiMedia, India, respectively. Chitosan supplied by HiMedia laboratories; Isooctane supplied by Sigma Laboratories, Hexanol supplied by SD-fine chemicals, and cetyltrimethylammonium bromide (CTAB) supplied by SD-fine chemicals. All Chemicals are used without further purification.

\section{Preparation of carboxymethyl chitosan (CMC)}

CMC was synthesized by our formerly reported protocol with a slight modification. Briefly, $1.0 \mathrm{~g}$ of chitosan is swelled in $40 \mathrm{ml}$ of $50 \%$ sodium hydroxide solution at $0^{\circ} \mathrm{C}$ overnight. Then, the chitosan is washed with isopropanol and dissolved in $30 \mathrm{ml}$ of isopropanol and the monochloracetic acid (3.0 g) was dissolved in isopropanol $(5 \mathrm{ml})$ and added into the reaction mixture dropwise for $20 \mathrm{~min}$ and reacted for $12 \mathrm{~h}$ at the $40^{\circ} \mathrm{C}$. Then, the reaction was stopped by adding $70 \%$ ethyl alcohol $(25 \mathrm{ml})$. The solid was filtered and rinsed in 70-90\% ethyl alcohol, and vacuum dried at room temperature. The resulted product was the sodium salt of CMC [17].

\section{Preparation of CMC NPs by ionic gelation method}

CMC NPs were synthesized by ion tropic gelation of CMC solution with calcium chloride solution. About $0.5 \%$ CMC solution was prepared in distilled water. To $5 \mathrm{ml}$ of this solution, $1 \mathrm{ml}$ of $1.5 \% \mathrm{CaCl}_{2}$ solution was added under constant stirring. The resulting NPs were purified by centrifugation for $10 \mathrm{~min}$ at $15,000 \mathrm{rpm}$ and lyophilized [18]. A diagrammatical representation is given in Fig. 1 for better insight into the development of NCs.

\section{Drug loading into CMC NPs}

EM $(1 \mathrm{mg} / \mathrm{mL})$ is poorly water-soluble drug was dissolved in $0.05 \%$ $10 \mathrm{ml}$ glacial acetic acid solution. $10 \mathrm{mg}$ CMC NPs were (per ml CMC NPs) introduced into the previously prepared drug solutions. The solution was shaken in an orbital shaker for $24 \mathrm{~h}$. The suspension was then centrifuged at 15,000 rpm min to separate the NPs from the solutions. The procedure was repeated 3 times to eliminate any unloaded eprosartan from the suspension. The amount of loaded eprosartan was measured spectrophotometrically at $234 \mathrm{~nm}$ with a UV-1700 spectrophotometer (Shimadzu). The drug loading content and entrapment efficiency were calculated according to the following equations.

\section{Weight of drug in

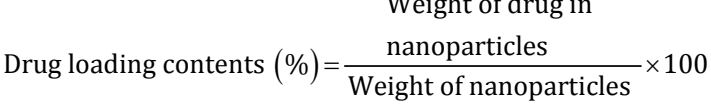 taken}

Drug entrapment efficiency $(\%)=\frac{\begin{array}{l}\text { Weight of drug in } \\ \text { nanoparticles }\end{array}}{\begin{array}{l}\text { Weight of } \\ \text { nanoparticles taken }\end{array}} \times 100$

Preparation of CS NPs using microemulsion/reverse micelle technique

Eprosartan loaded chitosan NPs were prepared using cetyltrimethylammonium bromide as a surfactant, isooctane as oil, 1-hexanol as cosolvent, eprosartan (concentration $5 \mathrm{mg} / \mathrm{ml}$ ) as the model drug, and chitosan-eprosartan solution as the aqueous phase. To prepare the reversed micelles, CTAB (used in a concentration of $5 \mathrm{mg} / \mathrm{ml}$ ), isooctane oil, and cosolvent were poured into a test tube. The chitosan (in concentration of $5 \mathrm{mg} / \mathrm{ml}$ ) in acetic acid solution, glutaraldehyde and eprosartan were then added to the mixture of CTAB and solvent under continuous stirring at room temperature.
NPs were formed in the presence of a surfactant. The system was stirred overnight to complete the cross-linking process, which the free amine group of chitosan conjugates with glutaraldehyde. The organic solvent is then removed by evaporation under low pressure. The formation of reversed micelles was inferred the mixed emulsion became transparent or semi-transparent. The completion of the reaction was recognized when the reverse micelle solvent becomes turbid. The yields obtained were the cross-linked chitosan NP and excess surfactant. The excess surfactant was then removed by precipitate with $\mathrm{CaCl}_{2}$ and then the precipitant was removed by centrifugation at $6000 \mathrm{rpm}$ for $15 \mathrm{~min}$. Finally, the obtained NPs were dried for $24 \mathrm{~h}$ in the air at the laboratory ambient temperature.

The eprosartan loaded chitosan NPs obtained from both the process were collected and compared for particle size, particle size distribution, zeta potential, scanning electron microscopy (SEM), transmission electron microscopy (TEM), and dissolution studies.

\section{Characterization}

\section{Particle size and zeta potential measurements}

Mean particle size and its distribution were determined using dynamic light scattering (DLS) technique. Zeta potential was evaluated by measuring electrophoretic mobility of the particle using a laserbased multiple angle electrophoresis analyzer. Eprosartan NPs were sufficiently diluted with deionized water to reduce the count rate up to 100-250 kcps and analyzed by employing Malvern Zetasizer (Nano ZS, Malvern Instruments, UK). Observations were recorded in triplicate for each sample at $25^{\circ} \mathrm{C} \pm 1^{\circ} \mathrm{C}$.

\section{Particle size measurement by SEM and TEM}

Particle's morphological characterization of eprosartan-NPs was executed by SEM and TEM. Samples for SEM studies were prepared by clinging the NPs on a double adhesive tape stuck to an aluminum stub and were coated with gold and palladium under an argon atmosphere utilizing a high-vacuum evaporator (Polaron SEM coating system). Samples were scanned through an electron microscope (EVO-50, ZEISS; UK) and photomicrographs were captured.

For TEM, a drop of optimized NPs suspension in aqueous solution was placed on a carbon film coated on a copper grid and freeze-dried. Then, NPs were observed at $80 \mathrm{kV}$ using TEM TECNAI G 20 S-TWIN (FEI, the Netherlands) instrument, Indian Institute of Toxicology Research, Lucknow.

UV and Fourier transform infrared (FTIR) studies of eprosartan CS NPs

The physical and chemical aggregation state of eprosartan was evaluated by UV and FTIR spectroscopy. The FTIR spectrums of eprosartan and drug-loaded chitosan NPs (freeze-dried) were obtained using spectrometer (Perkin Elmer). A total of $2 \mathrm{mg}$ of sample was mixed with $100 \mathrm{mg}$ of dry potassium bromide (KBr), and the mixture was ground into fine powder using a mortar before compressing into $(\mathrm{KBr})$ disk under a hydraulic press at 10,000 psi. Each $\mathrm{KBr}$ disk was then scanned at $4 \mathrm{~mm} / \mathrm{s}$ at a resolution of $2 / \mathrm{cm}$ over a wave number region of $400-4000 / \mathrm{cm}$ using IR solution (software ver. 1.10). The characteristics peaks of functional groups in the drug samples were determined and compared with pharmacopeial standards, and the

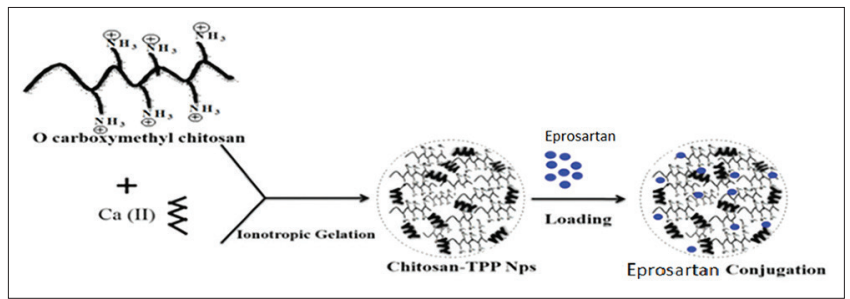

Fig. 1: Schematic representation of synthetic procedure of carboxymethyl chitosan-eprosartan 


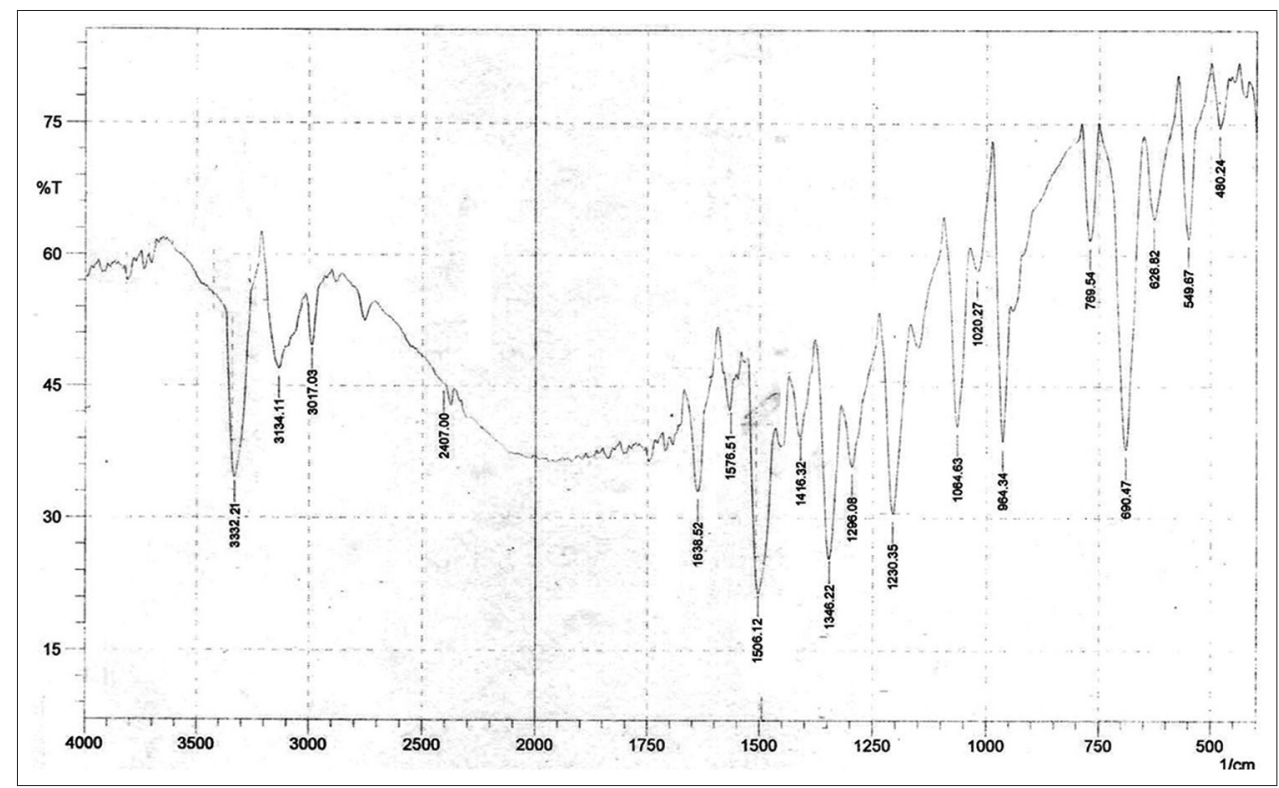

Fig. 2: Fourier transforms infrared spectrum of eprosartan mesylate

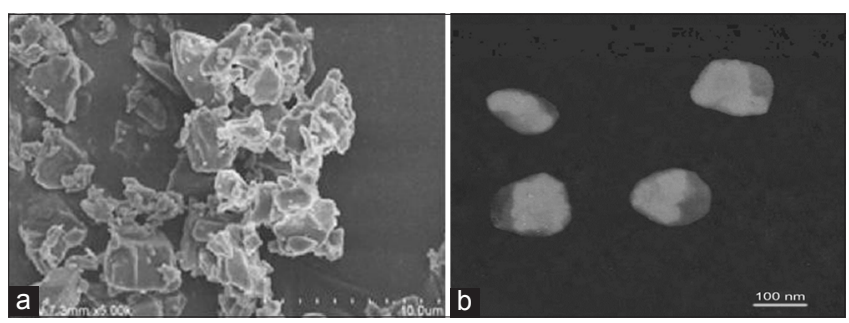

Fig. 3: (a) Scanning electron microscopy of eprosartan nanoparticles; (b) transmission electron microscopy of eprosartan nanoparticles

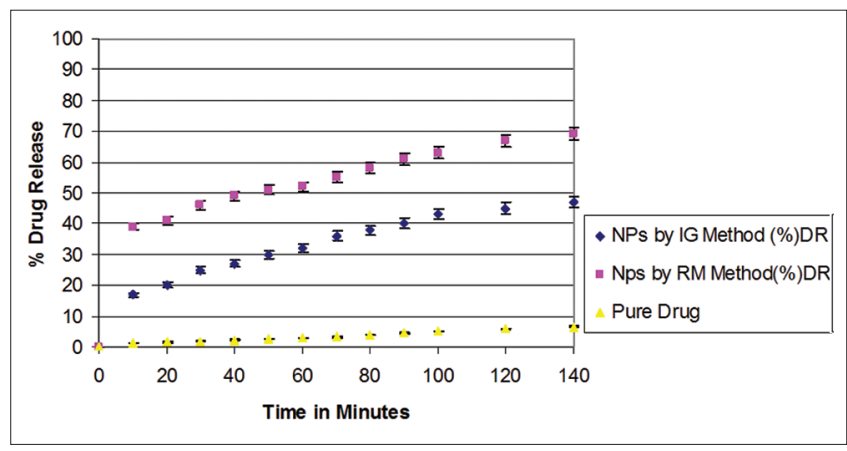

Fig. 4: In vitro release studies of eprosartan nanoparticles

possible interactions between drug and excipients were analyzed by obtained data. The electronic configuration of eprosartan NPs was elucidated by a UV spectrophotometer (UV 1700, Shimadzu, Japan). Lyophilized DTX-NCs were dispersed in TDW $(3.0 \mathrm{~mL})$ and scanned in the range from 200 to $400 \mathrm{~nm}$. The UV spectrum of pure eprosartan was taken after solubilizing it in methanol.

\section{Evaluation studies}

Dissolution studies

In vitro drug release study for optimized formulations of eprosartan loaded polymeric NPs was conducted in triplicate by equilibrium dialysis membrane method. The amount of drug release from the formulation was measured by a spectroscopic method. The experiment was performed by taking the specified volume of formulation in hermetically sealed dialysis membrane. The dialysis bag is suspended in $\mathrm{pH} 7.4$ phosphate buffer saline (PBS) at $37^{\circ} \mathrm{C}$ being stirred at $240 \mathrm{rpm}$ speed. Samples were collected at predetermined time points, and subsequently, media were replenished with pH 7.4 PBS after each sampling to maintain sink conditions.

\section{RESULTS AND DISCUSSION}

\section{FTIR analysis}

FTIR study was conducted to characterize any possible interaction between drug and CMCS NPs. The characteristic peaks at 3332.21 and $3134.11 / \mathrm{cm}(\mathrm{O}-\mathrm{H}$ stretching and N-H stretching vibrations), $2407.00 / \mathrm{cm}$ ( $\mathrm{C}=\mathrm{N}$ stretching vibration), and $1638.52 / \mathrm{cm}$ (corresponding to the $\mathrm{C}=\mathrm{C}$ ) confirm the formation of CMCS, as shown in Fig. 2.

\section{DLS}

The hydrodynamic diameter of eprosartan chitosan NPs prepared by reverse micellar technique is measured by DLS which is $80 \pm 10 \mathrm{~nm}$, whereas the eprosartan chitosan NPs prepared by ionic gelation method were $450 \pm 10 \mathrm{~nm}$. Results represent that there is a significant difference between the two types of NPs, which demonstrate that the NPs prepared by reverse micellar technique were significantly smaller in particle size. Zeta potential $\alpha$ is an electric potential of the shear plane attached to the moving particles in the medium, and it is generally utilized for the prediction of stability of nanosuspensions. The decrease in zeta potential value with a decrease in concentration of stabilizer may be attributed to the inability of stabilizer to prevent particle agglomeration at lower concentrations. The results of the optimized batches of eprosartanloaded nanosuspension prepared from both the processes depicted the zeta potential values of $35 \mathrm{mV}$ and $50 \mathrm{mV}$ of eprosartan chitosan NPs by ionic gelation process and microemulsion process, respectively, which suggested physically stable formulations were developed.

\section{Particle size measurement by SEM and TEM (Fig. 3)}

The SEM study reveals that the surface of the NPs was smooth and no aggregate were found from the sample used for SEM analysis. The particle size analysis done by TEM shows that the average particle size of the NPs was found to be 450 and $100 \mathrm{~nm}$ by ionic gelation and reverse micellar technique respectively. The study results are shown in Fig. 3.

\section{Dissolution studies}

The in vitro drug release pattern of NPs prepared by both the method against the pure drug is shown in Fig. 4. The solubility of the pure drug is very low because of which $<10 \%$ drug is released in $140 \mathrm{~min}$. 
According to the drug release pattern of NPs by IG method, there is a significant increase in percentage drug release as compared to the pure drug while the percentage drug release of NPs by RM method is increased significantly as compared to that of IG method. This increase in percentage drug release could be attributed to the increase in solubility of the drug when formulated as NPs. The percentage drug release pattern of both the NPs shows that the NPs prepared by RM method have increased solubility as compared to the IG method. The reason behind the enhancement in the solubility could be the reduction in particle size. According to Noyes Whitney equation, it has been observed experimentally that the reduction in particle size of a sparingly soluble material results in an increased rate of solution.

\section{Drug release pattern}

The percentage drug release pattern of both formulations was compared against that of pure drug. It shows that in $10 \mathrm{~min} \mathrm{39 \%}$ and $17 \%$ of the drug was released from the NPs made by RM method and microemulsion method, respectively, as compared to that of $1.3 \%$ of the pure drug. In 50 min almost more than half $51 \%$ of the drug was released from NPs by microemulsion method whereas only $2.5 \%$ of the drug was released from NPs containing pure drug. In $120 \mathrm{~min} 67 \%$ of the drug was released from NPs by microemulsion method whereas only $5.8 \%$ of drug release was shown by NPs with the pure drug.

\section{CONCLUSION}

The Chitosan NPs prepared by reverse micellar method were found to be more promising then ionic gelation method. The particle size of NPs obtained by the reverse micellar method was significantly reduced as compared to the other method. The reverse micellar method was found to be the best method for the preparation of eprosartan loaded chitosan NPs.

\section{ACKNOWLEDGMENT}

Authors would like to thank Amity University, Lucknow, for providing research facilities. CSIR-CDRI, Lucknow, is acknowledged for providing the support for research work.

\section{AUTHOR'S CONTRIBUTIONS}

UY, NH, and QR planned the study. UY and QR designed the research protocol. UY compiled the data. UY and QR formulate and interpreted the relevant data. UY drafted the manuscript. UY, NH, and QR critically revised the manuscript for intellectual content. All authors read and approved the final manuscript. UY, NH, and QR are guarantors of the paper.

\section{CONFLICTS OF INTEREST}

None of the author has any conflicts of interest in the context of this work.

\section{REFERENCES}

1. Anantha RG, Harish MN, Arthob NY, Keshavayya J, Venugopala RK. High performance liquid chromatographic analysis or determination of eprosartan mesylate in bulk drug. J Chem Pharma Res 2011;3:945-49.

2. Chandana OS, Sathis DK, Ravichandra B. Method development and validation of eprosartan mesylate and its imputies using reverse phase high performance liquid chromatography. Int J Curr Pharm Res 2016;8:49-53.

3. Rao ME, Suryakanta S, Shakti PM. Formulation design, optimization and characterization of eprosartan mesylate nanoparticles. Nanosci Nanotechnol Asia 2018;8:130-43.

4. Rewar S, Bansal BK, Singh CJ, Sharma AK. Approch for quantitative estmation of eprosartan mesylate by UV spectrophotometer. Int J Res Dev Pharm Lif 2014;3:1300-03.

5. Gassmann P, List M, Schweitzer A, Sucker H. Hydrosols-alternatives for the parenteral application of poorly water soluble drugs. Eur J Pharm Biopharm 1994;40:64-72.

6. Müller RH, Jacobs C, Kayser O. Nanosuspensions as particulate drug formulations in therapy. Rationale for development and what we can expect for the future. Adv Drug Deliv Rev 2001;47:3-19.

7. Dolenc A, Kristl J, Baumgartner S, Planinsek O. Advantages of celecoxib nanosuspension formulation and transformation into tablets. Int J Pharm 2009;376:204-12.

8. Jinno J, Kamada N, Miyake M, Yamada K, Mukai T, Odomi M, et al. Effect of particle size reduction on dissolution and oral absorption of a poorly water-soluble drug, cilostazol, in beagle dogs. J Control Release 2006;111:56-64.

9. Müller RH, Peters K. Nanosuspensions for the formulation of poorly soluble drugs: I. Preparation by a size-reduction technique. Int J Pharm 1998; 160:229-37.

10. Dong $\mathrm{Y}$, Ng WK, Shen S, Kim S, Tan RB. Preparation and characterization of spironolactone nanoparticles by antisolvent precipitation. Int J Pharm 2009;375:84-8.

11. Kesisoglou F, Panmai S, Wu Y. Nanosizing-oral formulation development and biopharmaceutical evaluation. Adv Drug Deliv Rev 2007;59:631-44.

12. Krishna SA, Amareshwar P. Preparation of bovine serum albumin loaded chitosan nanoparticle using reverse micelle method. Res J Pharm Biol Chem Sci 2011;2:837-46.

13. Abdol QM, Mohamed AO, Gamal M, Maghraby EL. Intestinal absorption of eprosartan mesylate from self emulsifying system and cyclodextrin complex. Int J Pharm Pharm Sci 2017;9:302-7.

14. Jassem NA, Rajab NA. Formulation and in-vitro evaluation of azilsartan medoxomil nanosuspension. Int J Pharm Pharm Sci 2017;9:110-9.

15. Krishna SA, Amareshwar P, Chakravarty P. Different techniques used for the preparation of nanoparticles using natural polymers and their application. Int J Pharm Sci 2011;3:45-50.

16. Graham B, Breezer AE. The relationship between particle size and solubility. Int J Pharm 1992;3:82.

17. Yadav U, Chowdhuri AR, Sahu SK, Husain N, Rehman Q. Formulation of nanoparticles of telmisartan incorporated in carboxymethyl chitosan for the better drug delivery and enhanced bioavailability Asian J Pharm Clin Res 2017;10:236-41.

18. Chen XG, Park HJ. Chemical characteristics of O-carboxymethyl chitosans related to the perpartion condition. Carbohydrate 2003;53:355-9. 